\title{
Beschreibung der sechs Nauplius-Stadien von Parastenocaris phyllura Kiefer, 1938 im Vergleich mit $P$. vicesima Klie, 1935 (Copepoda, Harpacticoida)
}

\author{
Thomas Glatzel \\ Arbeitsgruppe Zoomorphologie, Fachbereich Biologie, Universität Oldenburg, Postfach 2503, D-2900 \\ Oldenburg, Deutschland
}

Keywords: Copepoda, Parastenocaris phyllura, groundwater, nauplius stages, ontogeny

\begin{abstract}
Six non-overlapping size classes and morphologically distinct nauplius stages of Parastenocaris phyllura are studied using light microscopy. The nauplii have been reared in laboratory cultures. A comparative analysis of the morphology of the nauplius stages of $P$. phyllura and $P$. vicesima (cf. Schminke, 1982) is presented.
\end{abstract}

\section{Zusammenfassung}

Die sechs Nauplius-Stadien von Parastenocaris phyllura wurden im Labor gezüchtet. Mit Hilfe der Lichtmikroskopie wird die Morphologie der einzelnen Nauplien dokumentiert. Die Nauplius-Stadien von $\boldsymbol{P}$. phyllura werden mit den Nauplii von $P$. vicesima (siehe Schminke, 1982) verglichen und ihre Unterschiede herausgestellt.

\section{Einleitung}

Während Borutzky $(1925,1952)$ für Parastenocaris fünf Nauplius- und fünf Copepodid-Stadien angibt, beschreibt Schminke (1982) für Parastenocaris vicesima Klie, 1935 auch fünf NaupliusStadien und nimmt gleichzeitig an, daß die Art doch sechs Stadien durchläuft. Schminke zeichnet auch ein sechstes Nauplius-Stadium und behandelt den Nauplius 4/5 als einzelnes Stadium, weil keine morphologische Unterscheidung möglich war.

Chappuis (1916), Carter \& Bredford (1972) wie auch Sarvala (1977a, b) und Dahms (1987) zeigen mit den Untersuchungen der Entwicklung von lim- nischen Harpacticoiden, daß sechs NaupliusStadien die Regel sind. Eine genaue Untersuchung der Nauplius-Stadien des Grundwasserharpacticoiden Parastenocaris phyllura Kiefer, 1938 ermöglicht erstmalig einen Vergleich mit den Angaben von Schminke (1982) über $P$. vicesima.

\section{Material und Methoden}

Die hier untersuchten Nauplien von Parastenocaris phyllura stammen aus Laborzuchten des Autors. Die Elterntiere wurden mit Hilfe einer Membranpumpe aus einem geschlagenen Brunnen bei Walchum (Ems) und durch Ufergrabungen aus den Sandstränden des Großen Plöner Sees gesammelt und bei $8^{\circ} \mathrm{C}$ gezüchtet (siehe Glatzel, 1989, 1990).

Zur Untersuchung wurden die Nauplien in 5\% Formalin fixiert, in ein Gemisch aus Aqua dest. und dem Einbettungsmedium 'W15-Zeiss"' (wird nicht mehr hergestellt) überführt und nach dem Verflüchtigen des Aqua dest. in einem von Paraffin umgebenen W-15-Tropfen zu Dauerpräparaten verarbeitet. Die Zeichnungen wurden mit einem Interferenzkontrastmikroskop "Leitz Dialux 22 EB" unter Verwendung des Immersionsobjektives (100/1.32) und eines Zeichenspiegels angefertigt.

\section{Die Nauplius-Stadien von Parastenocaris phyllura}

Nauplius 1 (Fig. 1A)

Die Länge variiert zwischen 68-74 $\mu \mathrm{m}$, die Breite zwischen 44,5-51 $\mu \mathrm{m}$, gemessen an 15 Individuen. Der Körper ist dorsoventral abgeflacht, farblos, ein Nauplius-Auge fehlt. Das Labrum mißt etwa ein Drittel der Körperlänge, ist auf halber Länge tail- 

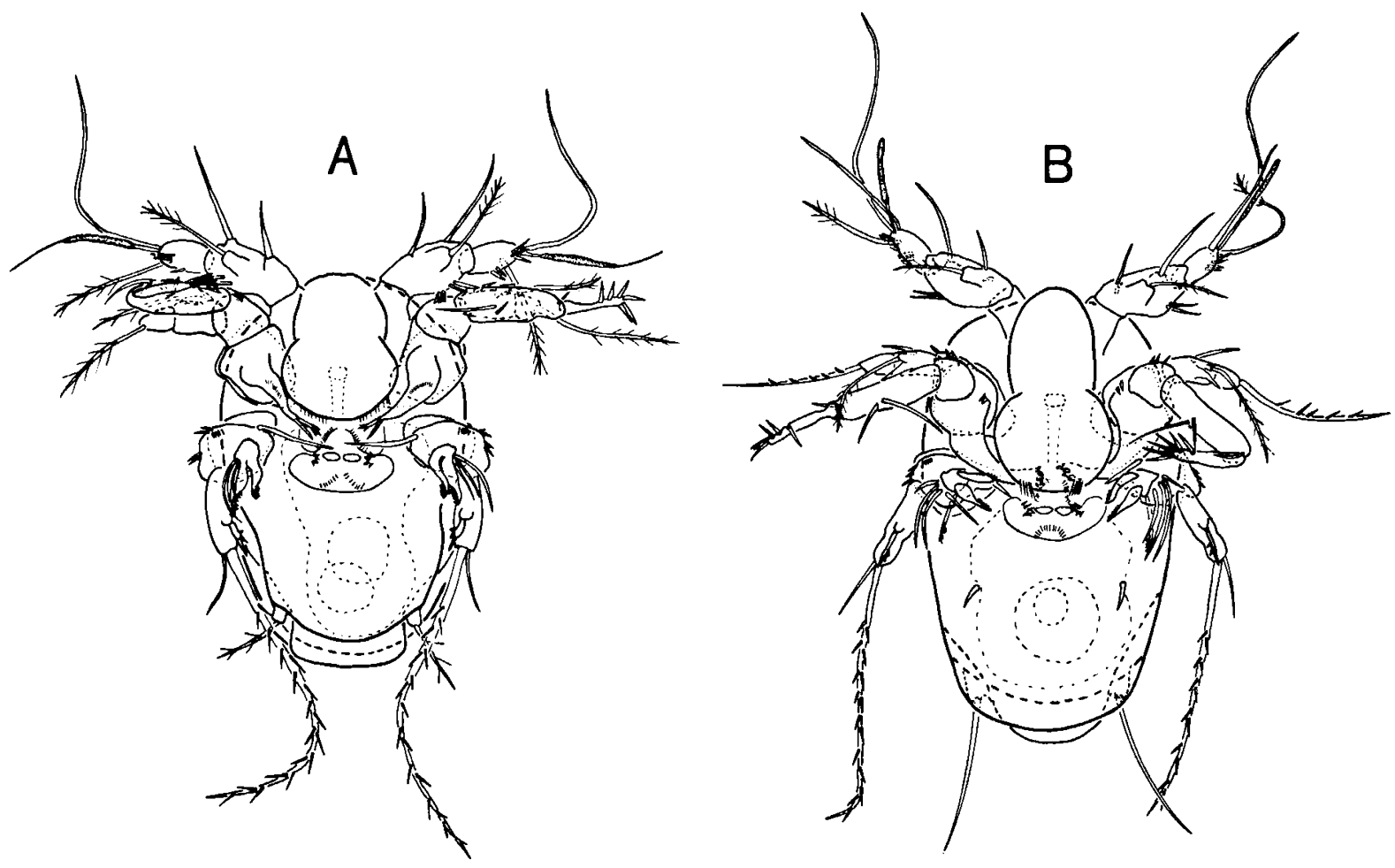

Fig. 1. Nauplius-Stadien von Parastenocaris phyllura. Ventralansichten: A, Nauplius 1, Länge $73 \mu \mathrm{m}$, Breite 44,5 $\mu \mathrm{m}$; B, Nauplius 2 , Länge $82 \mu \mathrm{m}$, Breite $49 \mu \mathrm{m}$.

liert und verjüngt sich etwas frontad. Sein fast kreisrunder Rand ist beidseitig jeweils mit 9 feinen Dörnchen besetzt. Unterhalb hiervon befindet sich der anteriore Rand des Mundfeldes, welcher beidseitig frontad je 1 konvexe Ausstülpung aufweist, deren Innenflanken mit feinen Härchen eingekleidet sind. Der caudale Bereich ist median mit zwei Börstchenreihen und zwei kleinen Plättchen besetzt. Beidseitig dieser Plättchen inserieren je 5 Dörnchen.

Das Analoperculum ist beinahe rechteckig geformt. Rechts und links von ihm entspringt auf einem Sockel je 1 befiederte Borste.

Antenna 1: 3-gliedrig; 1. Glied kurz und nackt; 2. Glied mit 2 Borsten entlang des Innenrandes, auf einem Sockel entspringt ventral 1 befiederte Borste und auf dem Außenrand 1 Reihe von 2 längeren Dörnchen; 3. Glied mit 1 langen Borste, 1 borstenartig auslaufenden gestielten Ästhetasken, 1 subterminalen Fiederborste und 2 Dörnchenreihen, die terminal und am medianen Außenrand inserieren.
Antenna 2: Coxa mit 1 Enditen, der sich distad verjüngt, bis zum Zentrum des Mundfeldes reicht und in 1 lateralen terminalen Borste mit einer Reihe von insgesamt 8 sich abwechselnden kleinen und kräftigen Zähnchen endet; Basis mit 1 Dörnchenreihe; Exopodit 2-gliedrig, 1. Glied mit 2 kurzen und 1 befiederten Borste, 2. Glied mit 1 terminalen befiederten Borste; Endopodit 1-gliedrig, 1. Glied mit 1 langen und 1 kurzen Borste am medianen Innenrand, apikal sitzt 1 prähensiler kräftiger Dorn, der distal mit 6 Fiedern besetzt ist und an dessen $\mathrm{Ba}-$ sis 1 kleine Borste entspringt.

Mandibel: Coxa dreieckig mit 1 langen Innenrandborste, die bis zur Mundfeldmitte reicht; Basis mit Dörnchenreihen; Exopodit 1-gliedrig, apikal mit 1 sehr langen befiederten und 1 kürzeren Borste, an der distalen Innenseite entspringt 1 dicke Borste, die 2 kurze und 1 längere Fieder trägt; Endopodit mit 1 kürzeren und 1 längeren Dorn, die jeweils an der distalen Innenseite einen "Kamm" von 5 Dörnchen tragen, an der äußeren Basis dieser 

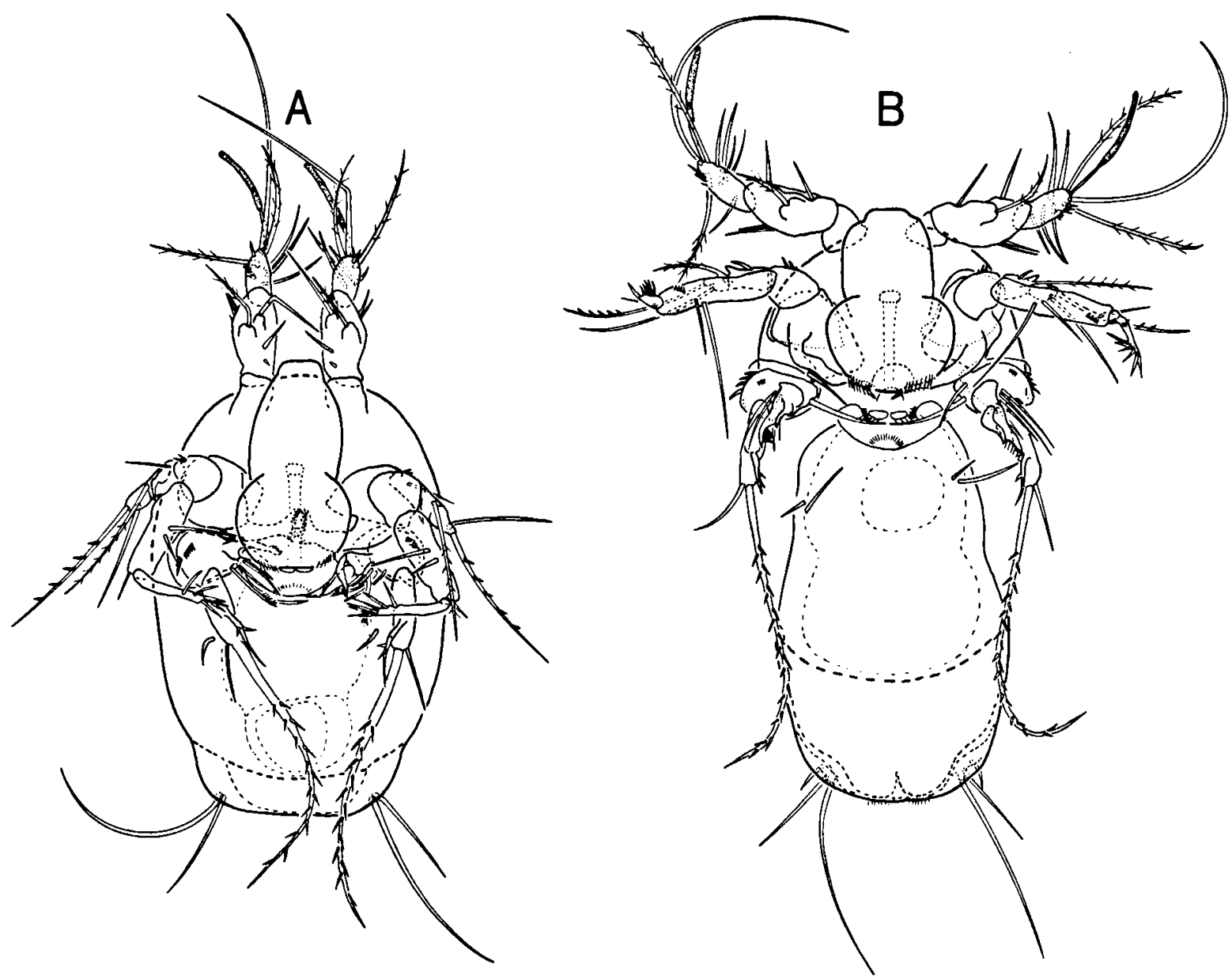

Fig. 2. Nauplius-Stadien von Parastenocaris phyllura. Ventralansichten: A, Nauplius 3, Länge $92 \mu \mathrm{m}$, Breite $59 \mu \mathrm{m}$; B, Nauplius 4 , Länge $122 \mu \mathrm{m}$, Breite $56 \mu \mathrm{m}$.

“Zange" entspringen 3 Borsten, die von außen nach innen kürzer werden.

\section{Nauplius 2 (Fig. 1B)}

Die Körperlänge beträgt zwischen 82-87 $\mu \mathrm{m}$, die Körperbreite zwischen 49-52 $\mu \mathrm{m}$, gemessen an 10 Individuen. Das Analoperculum ist rund.

Nauplius 2 unterscheidet sich in folgenden Merkmalen vom Nauplius 1:

Antenna 1: Zweites Glied mit 1 kleinen proximalen Borste; Endglied mit 1 zusätzlichen terminalen Borste.

Antenna 2: Coxa mit 1 Dörnchenreihe am Ur- sprung des Enditen; eine der beiden kurzen Borsten am 1. Glied des Exopoditen ist zum Dorn geworden.

Mandibel: Basis mit 1 inneren befiederten Borste und 1 weiteren kurzen Borste am Außenrand der 3 vorhandenen Borsten an der Basis des Endopoditen.

Maxilla 1: Durch 1 Borste vertreten.

Nauplius 3 (Fig. 2A)

Die Länge variiert zwischen 90-98 $\mu \mathrm{m}$, die Breite zwischen 52-59 $\mu \mathrm{m}$, gemessen an 10 Individuen. Das Analoperculum ist rund ausgebildet. 

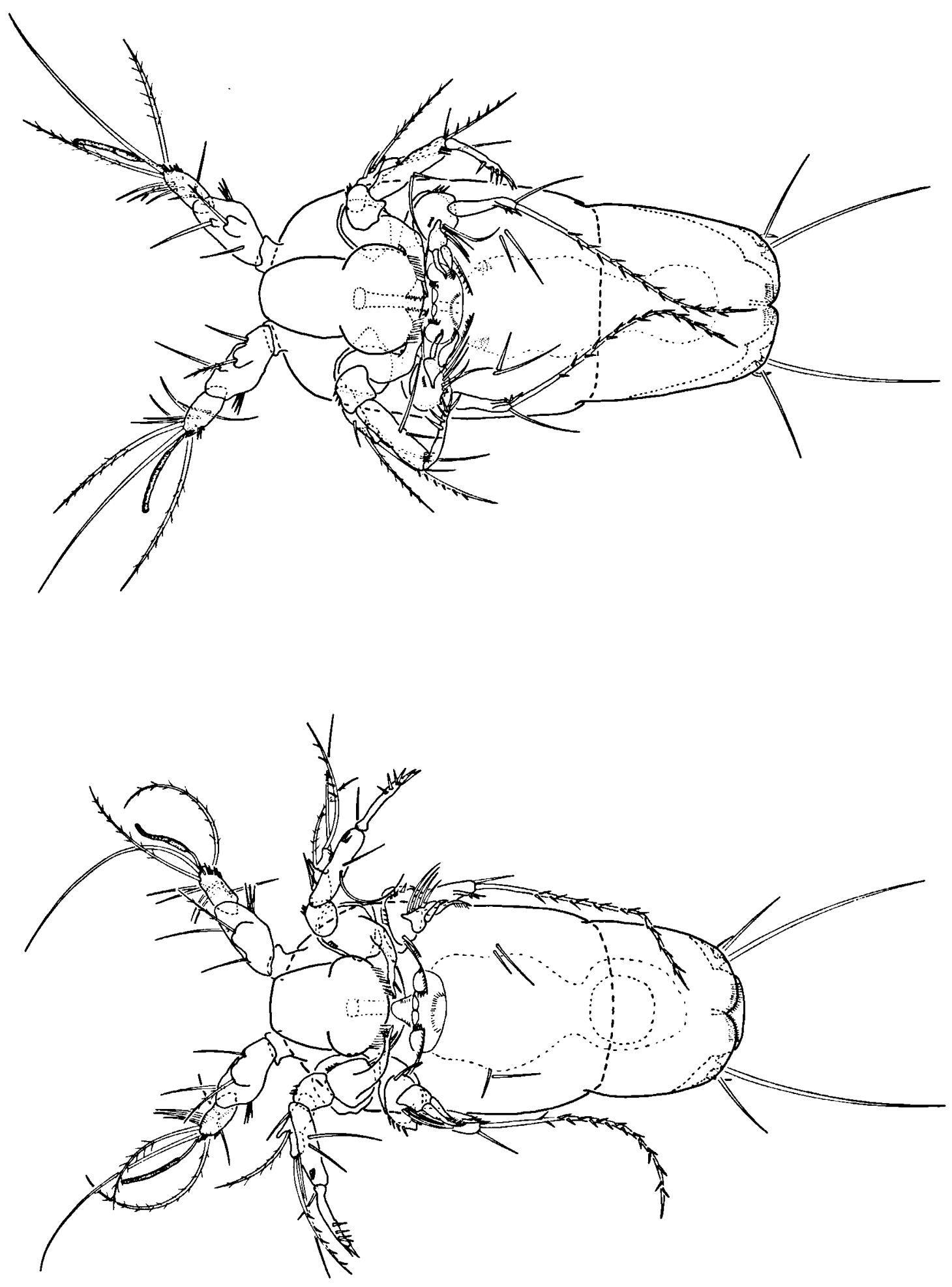
Nauplius 3 unterscheidet sich vom Nauplius 2 in folgenden Merkmalen:

Antenna 1: Endglied mit 3 weiteren Borsten; 2 der 6 Borsten sind befiedert.

Antenna 2: Endit läuft distal in 8 sich abwechselnde große und kleine Zähnchen aus, am terminalen Außenrand entspringt in kleinem Abstand 1 weiteres Zähnchen; Exopodit, 2. Glied mit 1 langen zusätzlichen distalen Borste.

Abdomen: Je 1 weitere Borste zu beiden Seiten des Analoperculums.

Nauplius 4 (Fig. 2B)

Die Länge und Breite variieren zwischen 108-121 $\mu \mathrm{m}$ und $54-56,5 \mu \mathrm{m}$, gemessen an 10 Individuen. Mit diesem Stadium tritt ein kräftiges Längenwachstum ein, so daß der Rückenschild nun in der Länge zurückbleibt. Das Analoperculum ist gerundet und der After ist mit feinen Härchen bewehrt.

Nauplius 4 unterscheidet sich in folgenden Merkmalen vom Nauplius 3:

Antenna 1: Mit 2 zusätzlichen Borsten am 3. Glied.

Antenna 2: Endopodit mit 1 distalen Dörnchenreihe.

Mandibel: Die lange befiederte Endborste reicht kaum noch über das Körperende hinweg.

Maxilla 1: Mit 1 weiteren kleinen Borste.

Abdomen: Beidseitig mit 1 zusätzlichen kleinen Borste neben dem Analoperculum.

\section{Nauplius 5 (Fig. 3)}

Die Länge variiert zwischen 122-129 $\mu \mathrm{m}$, die Breite zwischen $60-63,5 \mu \mathrm{m}$, gemessen an 7 Individuen. Dieser Nauplius ist jetzt schon 4 mal so lang wie sein Labrum.

Nauplius 5 unterscheidet sich vom Nauplius 4 in folgenden Merkmalen:

Antenna 1: Die am Außenrand liegende Dörnchenreihe des 2 . Gliedes besteht nun aus 3 längeren Dörnchen; 3. Glied mit 1 zusätzlichen Borste.

Antenna 2: Unterhalb des Enditenursprunges 1 weitere Borste, die bis in die Mitte des Mundfeldes reicht.

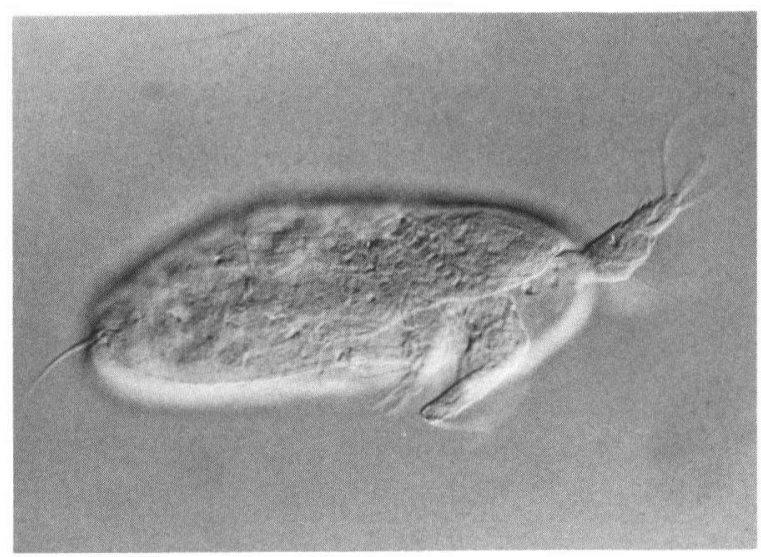

Fig. 5. Nauplius 6 von Parastenocaris phyllura. Lateralansicht Länge $139 \mu \mathrm{m}$.

Nauplius 6 (Fig. 4, 5)

Die Länge variiert zwischen 134-141 $\mu \mathrm{m}$, die Breite zwischen 61-65 $\mu \mathrm{m}$, gemessen an 15 Individuen. Unterhalb des Mundfeldes sind schon Borstenanlagen des Copepodiden 1 zu erkennen.

Nauplius 6 unterscheidet sich vom Nauplius 5 in folgendem Merkmale:

Mandibel: Die lange terminale Endborste erreicht nun nicht mehr das Ende des Abdomens.

\section{Diskussion}

In dieser Untersuchung wurden sechs NaupliusStadien von Parastenocaris phyllura nachgewiesen. Die Nauplius-Stadien wachsen im Verlauf der Entwicklung von ca. $70-140 \mu \mathrm{m}$. Schminke (1982) gibt ein Längenmaß von $62-120 \mu \mathrm{m}$ für die fünf Stadien von $P$. vicesima an (siehe Tabelle I). Der Schritt von seinem 4./5. Stadium bis zum 6. Stadium scheint um das Doppelte größer zu sein als die Wachstumsschritte bei den Nauplien von $P$. phyllura. Vermutlich ist das 6. Stadium von $P$. vicesima das eigentliche 5. Stadium und das wirkliche 6 . Stadium wurde nicht gefunden, oder der Nauplius 4 gleicht nicht dem Nauplius 5 und dieser wurde übersehen. Bei $P$. phyllura ist zwischen dem Nauplius 4 und 5 ein morphologischer Unterschied vorhanden, während der Unterschied zwischen dem Nauplius 5 und 6 nur im Größenwachstum zu fin- 
Tabelle I. Längenmaße $(\mu \mathrm{m}) \operatorname{der} 6$ Nauplius-Stadien von Parastenocaris.

\begin{tabular}{lcc}
\hline Stadium & $P$. phyllura & P. vicesima* \\
\hline Nauplius 1 & $68-74$ & 62 \\
Nauplius 2 & $82-87$ & 74 \\
Nauplius 3 & $90-98$ & 82 \\
Nauplius 4 & $108-121$ & $97 ?$ \\
Nauplius 5 & $122-129$ & $97 ?$ \\
Nauplius 6 & $134-141$ & 120
\end{tabular}

* Nach Schminke (1982)

den ist. Die sechs Nauplius-Stadien von $P$. phyllura verändern etwa alle $8-10 \mu \mathrm{m}$ in einer aufsteigenden Reihenfolge ihren Status.

Die Nauplius-Stadien von $P$. phyllura weisen gegenüber denen von $P$. vicesima (nach Schminke, 1982) folgende Unterschiede auf:

Nauplius 1:

Gestielter Ästhetask der Antenna 1 läuft borstenartig aus; Endit der Antenna 2 trägt terminal 8 Zähnchen, 1 kleines Außenranddörnchen und proximal eine Reihe von feinsten Dörnchen; Endopodit mit 1 kleinen apikalen Borste; Exopodit 2-gliedrig mit insgesamt 4 Borsten; Exopodit der Mandibel 1-gliedrig und 1 Dorn an der distalen Innenseite, der in 2 kurzen und 1 längeren Borste endet, apikal mit 1 längeren feinen Borste.

Nauplius 2:

Endopodit der Antenna 2 ohne 3 winzige Borsten in der Mitte der Innenseite des Gliedes; Basis des
Endopoditen der Mandibel jetzt mit 4 Borsten. Nauplius 3:

Basipodit der Mandibel ohne zusätzliche Borste. Nauplius 4:

Endborste des Endopoditen der Antenna 2 ohne siebten Dorn.

Nauplius 5:

Zweites Glied der Antenna 1 mit 1 zusätzlichen kleinen Borste; 3. Glied mit 1 weiteren kleinen proximalen Borste; unterhalb des Enditenursprunges der Antenna 2 eine lange Borste, die bis in die Mitte des Mundfeldes reicht.

Nauplius 6:

Nauplius $20 \mu \mathrm{m}$ länger; mittleres Glied der Antenna 1 mit 6 Borsten, keine Borstenreduktion; prähensile Endborste des Endopoditen der Antenna 2 nur mit 6 Fiedern; Exopodit 2-gliedrig; Exopodit der Mandibel 1-gliedrig.

Es ist trotz der beschriebenen Unterschiede schwierig zu sagen, ob der Nauplius 6 von $P$. vicesi$m a$ nun dem Nauplius 6 oder 5 von $P$. phyllura entspricht. Beachtet man aber die Längen- und Breitenunterschiede der beiden Stadien, so ist es eher wahrscheinlich, daß der Nauplius 6 von $P$. vicesima dem Nauplius 5 von $P$. phyllura entspricht. Nimmt man aber an, daß die beiden Nauplius-Stadien 6 einander entsprechen, so würde der Nauplius 5 von $P$. vicesima fehlen, und die große Wachstumsspanne von gut $20 \mu \mathrm{m}$ wäre dann gefüllt. Unterstützt wird diese Annahme von der Tatsache, daß die beiden Nauplius-Stadien 4 als erster Nauplius

Tabelle II. Unterschiede der Nauplius-Stadien von Parastenocaris (A1 = Antenna 1; A2 = Antenna 2; Md = Mandibel).

\begin{tabular}{lll}
\hline & $P$. phyllura & $P$. vicesima* \\
\hline A1, Nauplius 6 & 6 Borsten & 4 Borsten \\
A2, Nauplius 1-6 & Endit mit 8 Zähnchen & Endit mit 12 Zähnchen \\
A2, Nauplius 6 & Exopodit 2-gliedrig & Exopodit 3-gliedrig \\
A2, Nauplius 1-6 & Endopodit mit 2 terminalen & Endopodit mit 1 terminalen Borste, ab \\
& Borsten, eine davon mit 6 kräftigen & Nauplius 4/5 mit 7 kräftigen Fiedern \\
A2, Nauplius 5-6 & Fiedern & Coxa ohne Borste \\
Md, Nauplius 1-6 & Coxa mit 1 langen Borste & ab Nauplius 3 Basipodit mit 1 zusätzlichen \\
Md, Nauplius 1-6 & ab Nauplius 3 Basipodit ohne & Borste \\
zusätzliche Borste & am äußeren Endopoditen immer 3 Borsten \\
Md, Nauplius 1-6 & ab Nauplius 2 am äußeren Endopoditen & Exopodit ab Nauplius 6 3-gliedrig, Innen- \\
& Exopodit immer 2-gliedrig, Innen- & Exten \\
\end{tabular}

* Nach Schminke (1982) 
jetzt mit drei Borsten am hinteren Ende ausgestattet sind.

Das allgemeine Längenwachstum kann auch nur indirekt verglichen werden, da der Nauplius 1 bei $\boldsymbol{P}$. vicesima nur mit $62 \mu \mathrm{m}$ angegeben ist, und keine Aussage darüber gemacht wird, wie alt dieses Individuum war. Bei der kleinsten Längeneinheit vom Nauplius 1 bei $P$. phyllura handelt es sich in mehreren Fällen um nur wenige Stunden alte Tiere.

Wie schon die Auflistung der Unterschiede der Nauplien beider Arten zeigt, kristallisieren sich doch einige auffällige und artspezifische Merkmale heraus, die in Tabelle II zusammengetragen sind.

\section{Danksagung}

Herr Prof. Dr. H.K. Schminke unterstützte diese Arbeit mit fachlichen Diskussionen und kritischen Anmerkungen. Meiner Frau Jutta und meinen Eltern danke ich herzlich für die immer bereitstehende Logistik.

Der Niedersächsische Minister für Wissenschaft und Kunst finanzierte diese Arbeit.

\section{Literatur}

Borutzky, E.V., 1925. Die Larven der Süßwasserfauna. Trudy kosin. biol. Sta., 3: 49-61.
Borutzky, E.V., 1952. Freshwater Harpacticoida. In: Fauna SSSR III, (4): 1-389 (translated in: Fauna USSR, Israel Program for Scientific Translation, Jerusalem, 1964).

Carter, M.E. \& J.M. Bradford, 1972. Postembryonic development of three species of freshwater harpacticoid Copepoda. Smithson. Contr. Zool., 119: 1-26.

Chappuis, P.A., 1916. Viguierella coeca Maupas. Revue suisse Zool., 24 (8): 521-564.

Dahms, H.U., 1987. Die Naupliusstadien von Bryocamptus pygmaeus Sars, 1862 (Copepoda, Harpacticoida). Drosera, 1: 47-58.

Glatzel, Th., 1989. Die Grundwasserfauna NordwestNiedersachsens sowie Untersuchungen zur Morphologie und Biologie an Vertretern der Chappuisiidae und Parastenocarididae (Copepoda, Harpacticoida): 1-172 (Dissertation, Universität Oldenburg).

Glatzel, Th., 1990. On the biology of Parastenocaris phyllura Kiefer (Copepoda, Harpacticoida). Stygologia, 5 (3): 131-136.

Sarvala, J., 1977a. The naupliar development of Bryocamptus zschokkei (Copepoda, Harpacticoida). Ann. Limnol., 13 (2): 115-131.

Sarvala, J., 1977b. The naupliar development of six species of freshwater harpacticoid Copepoda. Ann. zool. fenn., 14: 135-161.

Schminke, H.K., 1982. Die Nauplius-Stadien von Parastenocaris vicesima Klie, 1935 (Copepoda, Parastenocarididae). Drosera, 1: 101-108.

Eingegangen am 11. Februar 1991 